\title{
Das Staunen vor der Schöpfung: «Tota substantia», «calidum innatum», «generatio spontanea» und atomistische Formenlehre bei Daniel Sennert
}

\author{
Michael Stolberg
}

\section{SUMMARY}

A thorough study of the system of a philosophy of nature, elaborated by the wellknown German physician Daniel Sennert (1572-1637). At the beginning of the modern era, Sennert developed a new and very complex concept of the relations between matter and the guiding principle active in it, the Aristotelian form.

\section{ZuSAMMENFASSUNG}

Daniel Sennert (1572-1637) hat einen umfassenden und hochdifferenzierten naturphilosophischen Erklärungsansatz vorgelegt, der weit über das Wiederaufgreifen der antiken Atomistik hinausging und dessen Leistungsfähigkeit und Originalität bisher nur unzureichend gewürdigt wurde. Dieser Beitrag zeichnet die wesentlichen Züge und zentralen Problemstellungen von Sennerts Naturphilosophie nach und ortet ihre Wurzeln in einem letztlich religiös inspirierten und zugleich stark empirisch ausgerichteten Naturverständnis. Ein weiter Bogen führt dabei von der schon in den wenig bekannten Frühschriften angelegten Umdeutung der alten medizinischen Lehre von der "tota substantia» und vom «calidum innatum» bis hin zu einem radikal neuen Verständnis der "generatio spontanea», der Struktur der Materie und ihres Verhältnisses zur Form als Quelle ihrer Eigenschaften und Wirkkräfte.

Der Wittenberger Professor Daniel Sennert (1572-1637) war einer der bekanntesten und erfolgreichsten Autoren seiner Zeit. Seine Institutiones medicinae $^{1}$ zählten dank ihrer strengen Gliederung und ihres kompilatorischen Charakters bis ins 18. Jahrhundert hinein zu den beliebtesten medizinischen Lehrwerken. Besondere Beachtung hat sein Versuch gefunden, in De 
chymicorum cum Aristotelicis et Galenicis consensu et dissensu ${ }^{2}$ paracelsistische Konzepte in das Gedankengebäude der galenischen Medizin zu integrieren ${ }^{3}$. Mehr noch als viele andere zeitgenössische Mediziner verstand sich Sennert aber zugleich als Naturphilosoph und warnte ausdrücklich davor, die Naturphilosophie als ein blosses «tirocinium» der Medizin geringzuschätzen ${ }^{4}$. Die Geschichtsschreibung hat sich hier vor allem auf Sennerts Bedeutung für die Geschichte der Atomistik und der Lehre von den chemischen Verbindungen konzentriert ${ }^{5}$. Doch Sennerts Erklärungsanspruch war weit umfassender. Das ist in der Geschichtsschreibung bisher nicht ausreichend gewürdigt worden ${ }^{6}$. Unter Beibehaltung der Grenze zwischen Beseeltem und Unbeseeltem und unter Verwendung und Umdeutung traditioneller medizinischer Konzepte zielte er auf eine einheitliche Interpretation der Naturkörper und ihrer Erscheinungen im Lebendigen wie in der unbeseelten Materie. Dieses Bemühen nachzuzeichnen, macht sich der folgende Beitrag zur Aufgabe.

\section{Formenlehre und «tota substantia»}

Ausgangspunkt und Grundlage von Sennerts Entwurf ist eine Haltung des Staunens, einer letztlich religiös inspirierten Ehrfurcht vor der Vielfalt und Komplexität der Schöpfung und der wundersamen und je spezifischen Eigenart der Dinge in der Natur ${ }^{7}$. Vor dem Hintergrund der vorherrschenden aristotelischen Naturphilosophie führt ihn diese Haltung unmittelbar in die Auseinandersetzung um eines der zentralen Probleme der RenaissancePhilosophie, die Interpretation des Mischungsvorgangs. Welches, so die Grundfrage, ist die Quelle der je spezifischen Eigenschaften und Wirkkräfte der Naturkörper, und wie kann aus der blossen Mischung der Elemente etwas wesenhaft Neues hervorgehen? Sennert verwahrt sich strikt gegen Versuche, den Vorgang des Werdens komplexer Naturdinge durch die Annahme zu erklären, die Form des Werdenden werde hier aus der Potenz in den Akt überführt. Niemals könne die Mischung der Elemente etwas anderes hervorbringen, als das, was in den Elementen sei $^{8}$. Doch auch die zufällige räumliche Anordnung kleinster Teilchen im Sinne der antiken Atomistik scheint ihm keine befriedigende Erklärung für die Komplexität und Regelhaftigkeit in der Natur zu bieten. Vielmehr könnten die höheren Wirkvermögen der komplexen Naturkörper nur von entsprechenden überelementaren Formen ihren Ausgang nehmen, die keinesfalls als potentielle unter der Herrschaft 
der niedrigeren elementaren Formen vorstellbar seien. Der Vielfalt der Naturkörper und ihrer Wirkvermögen entspreche eine Vielfalt spezifischer, überelementarer Formen ${ }^{9}$.

Sennerts Antireduktionismus und seine Konzentration auf den dynamischen Aspekt der Form als Quelle der je spezifischen Qualitäten und Wirkvermögen der Naturkörper findet sich bereits in seinen frühen medizinischen Abhandlungen angelegt, in von ihm selbst verfassten Doktorthesen, die seine Studenten zur Erlangung der Doktorwürde verteidigen mussten. Dabei geht es zunächst vor allem um die spezifischen Wirkungen von Arzneimitteln und um die vielfältigen und sonderbaren Symptome bei kontagiösen Krankheiten, Pestfiebern und Vergiftungen. Im Anschluss an Jean Fernel fragt Sennert: «Gibt es Krankheiten der ganzen Substanz oder der Form [...]? ${ }^{10}$ » Fernels ontologischen Krankheitsbegriff lehnt er ab. Krankheiten beträfen nicht die essentielle Konstitution, bei der keine «intensio» oder «remissio» der Qualitäten möglich sei, sondern sie seien akzidentell ${ }^{11}$. Wohl aber sieht er in der Erfahrungswelt viele Beispiele für die besonderen okkulten Wirkvermögen bestimmter Stoffe auf den menschlichen Körper, die nicht von den Formen der Elemente, sondern allein von höheren überelementaren Formen ihren Ausgang nehmen könnten und die allgemein als «a tota substantia» bezeichnet würden. Ihnen entsprächen bestimmte Krankheiten, die man insofern als Krankheiten der ganzen Substanz bezeichnen könne ${ }^{12}$. Konkret diskutiert er das etwa anhand der purgierenden Wirkungen von Kathartika ${ }^{13}$. Deren Fähigkeit, gezielt den schädlichen Saft aus dem Körper zu entfernen, sei mit der anziehenden Fähigkeit elementarer Wärme nicht ausreichend erklärbar. Es müsse sich um ein spezifisches Anziehungsvermögen handeln, das allein aus einer höheren spezifischen Form, aus der ganzen Substanz und deren Beziehung zu dem betreffenden Saft hervorgehen könne. Entschieden wehrt er sich gegen Vorwürfe, die Rede von okkulten Qualitäten und ganzer Substanz solle nur das eigene Nichtwissen verdecken. Vielmehr machten sich jene der Tollkühnheit schuldig, die die Geheimnisse der verborgensten Naturdinge durchschauen und an die Stelle verborgener aber wahrer Manifeste falsche Qualitäten setzen wollten ${ }^{14}$. Die Erfahrung zeige, dass «allen Dingen gewisse höhere Eigenschaften eingegeben sind, durch welche sie, was ihnen jeweils eigen ist, durch die Natur und innewohnende Bewegung der Form bewirken [...]» ${ }^{15}$.

In den Institutiones medicinae von 1611 werden diese Thesen dann weiter entwickelt und spezifiziert ${ }^{16}$. Unter Berufung auf Galen führt Sennert hier ein wesentliches, in der Folgezeit vielfach wiederholtes Argument für die 
Existenz überelementarer Qualitäten und entsprechender Formen an: ihre Fähigkeit, anders als die elementaren Qualitäten unabhängig von ihrer Masse selbst in kleinster Menge grösste Wirkungen zu erzielen ${ }^{17}$. Die Giftmenge beim Stich des Skorpions sei so klein, dass sich in seinem Stachel nicht einmal ein Loch entdecken lasse, und seine Folgen doch verheerend ${ }^{18}$. Analoges gelte für die Kontagien ${ }^{19}$ :

«[...] was mitgeteilt wird, ist etwas Widernatürliches, [...] ein gewisser Körper, gleichsam ein Samen [...] Zur Natur dieses Samens aber gehört es, dass er jene okkulte Qualität enthält, die in dem Körper, von dem er aufgenommen wird, eine sogenannte Krankheit der ganzen Substanz hervorrufen kann : weshalb auch in einer winzigen Menge eine grosse Kraft des Kontagiums ist. Während nämlich die elementaren Qualitäten, damit sie viel bewirken, auch viel Materie brauchen, haben diese [okkulten] Qualitäten, da sie ja Parteigänger der Form sind, auch in einem winzigen Quantum grosse Kraft, und eine winzige Menge eines solchen Samens genügt, um den ganzen Körper zu infizieren.»

Weitere Belege für die Existenz und Wirkweise überelementarer Qualitäten findet Sennert unter anderem in spezifischen Sympathiebeziehungen, wie sie im Falle der Kontagien am unterschiedlich ausgeprägten Befall verschiedener Individuen, und bei bestimmten Giften und Heilmitteln an der gezielten Affektion bestimmter Körperteile erkennbar würden ${ }^{20}$.

\section{Das «calidum innatum»}

Der Versuch, die spezifischen Wirkvermögen insbesondere von Arzneimitteln auf die «tota substantia» im Sinne einer überelementaren Form zurückzuführen, führt unmittelbar zu der Frage nach der materiellen Verankerung dieser okkulten Qualitäten und nach ihrer Beziehung zu der Seele der Pflanzen oder Tiere, aus denen viele Arzneimittel gewonnen werden. Sennert stellt klar, dass die betreffende im Arzneimittel über ihre okkulten Qualitäten wirksame überelementare Form keinesfalls mit der Seele der Pflanze oder des Tiers identisch sein könne, da diese ja bei toten Pflanzen und Tieren definitionsgemäss abwesend $\operatorname{sei}^{21}$.

Wenn aber diese überelementare Form sich auch nicht der blossen Potenz der nach dem Tod verbleibenden elementaren Materie verdanken soll, wo kommt sie dann her? Letztlich betrifft diese Frage nicht nur die Arzneimittel, sondern alle Naturkörper mit überelementaren Formen und Wirkvermögen. Die neoplatonisierende Annahme eines Zustroms der Form von einer äusseren Formenquelle her lehnt Sennert entschieden ab. Ein göttlicher 
Eingriff in die Schöpfung ist ihm nur als Wunder vorstellbar, und die Lehre vom steten Wirken einer «anima mundi», einer «colchodea» ${ }^{22}$, oder eines sonstigen himmlischen Formengebers scheint er aus einem immanentistischen Naturverständnis heraus nicht als akzeptabel empfunden zu haben. Damit bleibt aber im Grunde nur eine Möglichkeit offen. Die betreffende Form muss sich in actu schon im lebenden Körper befunden haben, ehe dieser verging, ohne aber mit der Form des vergangenen Körpers - im Falle der Pflanzen und Tiere wäre das die Seele - identisch zu sein.

Das erscheint im Rahmen der herkömmlichen aristotelischen Formenlehre als eine widersinnige Behauptung ${ }^{23}$. Denn wo die Form die Wesenheit, das So-Sein der jeweiligen Naturkörper bestimmt, können nicht zwei verschiedene Formen zugleich hierfür verantwortlich sein. Zentrales Verdienst der aristotelischen Potenz-Akt-Lehre war es ja gerade, einen Ausweg aus diesem Dilemma gewiesen zu haben. Sennert aber umgeht diese Schwierigkeiten, indem er, der Bildlichkeit hierarchischer politischer Herrschaftsverhältnisse folgend, in den komplexen Naturdingen und Lebewesen eine Formenhierarchie konstruiert ${ }^{24}$. Die höchste, herrschende Form, im Falle der Tiere und Pflanzen also die Seele, bedient sich der untergeordneten Formen für ihre Zwecke. Letztere sind

«[...] für sich betrachtet auch wahre Formen, die ihre Materien formen, aber bezüglich der Seele und der spezifischen Form stehen sie in dem Verhältnis einer unmittelbaren Materie.» ${ }^{25}$

Wenn aber die herrschende Form untergeht und die von ihr vermittelte Einheit zerfällt,

«[...] bleiben diese Formen eine Zeit lang bestehen und fungieren als Formen und sind die Urheber der Vermögen und Funktionen, die in vielen Dingen, die nicht mehr leben, vorgefunden werden und dennoch nicht aus den Qualitäten oder Formen der Elemente entspringen können.» ${ }^{26}$

An diesem Punkt des Argumentationszusammenhangs nimmt Sennert eines der ältesten physiologischen Konzepte der abendländischen Medizin in seine Argumentation auf, die Lehre von der eingepflanzten oder eingeborenen Wärme und die eng damit verbundenen Vorstellungen vom «spiritus innatus» und vom «humidum radicale» ${ }^{27}$. Die vielfältigen Wirkvermögen, die die eingeborene Wärme als Instrument der Seele in den Ernährungs- und Fortpflanzungsvorgängen zu erkennen gebe, liessen keinen Zweifel daran, 
dass sich ihr Wirken nicht auf die elementaren Qualitäten zurückführen lasse ${ }^{28}$. Diese Wärme ist bei Sennert nicht blosses Wirkvermögen im Dienst der Seele. Es handle sich vielmehr um einen Stoff, um ein «calidum» ${ }^{29}$. In dieser Gestalt aber eignet sich das Konzept des «calidum innatum» hervorragend als Erklärungsschlüssel für die angesprochenen Medikamentenwirkungen. Nach dem Tod von Pflanze oder Tier und dem damit verbundenen Herrschaftsverlust der Seele übernimmt, Sennert zufolge, deren bisheriges stofflich verankertes Instrument, das «calidum innatum», seinerseits die Herrschaft. Es ist Quelle und materielles Substrat der überelementaren, heilenden Wirkvermögen.

Sennert weitet die Lehre vom «calidum innatum» freilich zumindest vorübergehend weit über den Bereich der Physiologie hinaus aus. Insofern ihm ein wichtiger Teil der arzneilichen Wirkkräfte auf spezifische Sympathie-Antipathie-Beziehungen zu verweisen scheint, liegt es nahe, im «calidum innatum» oder in einem Analogon des «calidum innatum» in den Nicht-Beseelten überhaupt die Quelle jeglicher Sympathie- oder Antipathie-Beziehungen zu sehen, von denen der Sennertsche Kosmos durchdrungen ist ${ }^{30}$. Sennert sieht die Dinge der Natur von etwas überelementarem «Spirituösen» durchwirkt ${ }^{31}$. Die Bezeichnungen «calidum innatum» verdanke der «spiritus» im Grunde nur seiner auffälligen Wärme in den Lebewesen. Es sei

«[...] nicht allein die Wärme Werkzeug der Seele, sondern jener ganze Körper, der mit dem Namen eingepflanzte Wärme oder «spiritus» bezeichnet wird, und der nicht nur mit Wärme, sondern auch mit anderen höheren Qualitäten begabt ist.» ${ }^{32}$

Insbesondere den Gemmen, Steinen und Mineralien schreibt Sennert einen solchen dem «calidum innatum» analogen «spiritus» $\mathrm{zu}^{33}$.

Einen entscheidenden und mehrfach hervorgehobenen Beleg für die Existenz einer solchen besonderen überelementaren Materie findet Sennert in den Lehren und Laborerfahrungen der Alchimisten. Ihnen gelinge es, den wirksamen heilenden Bestandteil von pflanzlichen oder tierischen Stoffen von dem unwirksamen Rest durch Destillation, Filterung und andere Trennverfahren zu isolieren und somit das «calidum innatum» sichtbar zu machen $^{34}$ :

«Wenn nämlich durch Kochen oder Aufguss der Teil der Substanz, in dem die Kraft des Purgierens ist, abgetrennt und extrahiert wird, wird ein nützliches Medikament zubereitet; der restliche Körper aber bleibt gänzlich unwirksam zurück.» 
Freilich bleibt immer noch - zumal für die Nicht-Beseelten - die Frage offen, wie denn diese materiell verankerte überelementare Form mit ihren erstaunlichen Wirkvermögen überhaupt in den betreffenden Naturkörper hatte Eingang finden können, um nach dem Vergehen von dessen spezifischer Form ihrerseits die Herrschaft übernehmen zu können. Analog seiner Haltung in der Formenlehre lehnt Sennert gegen Jean Fernel einen stetigen Zustrom von überhimmlischer Wärme oder «spiritus» strikt ab. Das «calidum innatum» fliesst nicht etwa im Augenblick der Empfängnis in das Kyema ein, sondern es wird wie die Seele von Generation zu Generation weitergegeben und geht somit letztlich auf die göttliche Erschaffung der Welt zurück. Die Bildlichkeit der Fortpflanzung wird aber auch paradigmatisch für die Erklärung des Ursprungs überelementarer Formen und Wirkvermögen in den nicht-beseelten Naturdingen. Sennert erkennt im biblischen Schöpfungsbericht einen entscheidenden Wissensvorsprung des christlichen Abendlandes vor der aristotelischen Lehre von der Ewigkeit der Welt ${ }^{35}$ :

«Richtiger lehren die heiligen Schriften uns, dass alle Dinge ihre Formen, durch die sie sich von einander unterscheiden und wirksam sind, aus dem ersten Schöpfungsakt haben und dieselben durch den Willen des allmächtigen Gottes bis heute bewahren.»

«Gott hat nämlich als Grundlage des Werdens der Dinge einen Samen jedes Dinges gesetzt, durch welches sich die Werdensfähigen fortpflanzen, und hat das Ganze selbst am Anfang gemischt; und so wird der Körper, der den Dingen von Anfang an eingegeben wurde, im Werden weitergegeben und von jenen Prinzipien, die anfangs gegründet wurden, bis zum heutigen Tag konstituiert, beherrscht und bewahrt.» ${ }^{36}$

Die Spezifität der Einzeldinge und ihre Wirkvermögen, so zeigt sich, gehen nicht auf die Mischung von Elementen oder auf das zufällige Zusammentreffen von Atomen, sondern letztlich auf den göttlichen Schöpfungsakt zurück. Die Formen bestehen zusammen mit ihrem zugehörigen Substrat seit dem Anfang der Welt.

Damit wird das traditionelle Problem der «mistio» buchstäblich auf den Kopf gestellt. Die Form des Werdenden ist nicht mehr Resultat, sondern Ursache des Mischungsvorgangs und seines spezifischen Ablaufs. Sie ordnet von kleinen Materiequanten ausgehend die umgebende Materie lenkend und organisierend ihrem Herrschaftsbereich unter. Die vielfältigen Formen der Naturdinge entstehen also nicht neu, sind stets schon vorhanden und vermehren sich allenfalls so, wie eine Kerzenflamme an eine zweite Kerze weitergegeben werden kann, ohne dass die erste verlöscht. 


\section{Generatio spontanea}

Diese Auffassung hat weitreichende Konsequenzen für jenes damals vieldiskutierte naturphilosophische Grundproblem, dem Sennert einen grossen Teil seines letzten Werks, der Hypomnemata physica ${ }^{37}$, widmet, der Frage nach dem Wesen der Urzeugung, der «generatio spontanea» ${ }^{38}$. Seiner antireduktionistischen Grundhaltung entsprechend, wendet sich Sennert auch hier strikt gegen Versuche, die Entstehung von Würmern und Insekten aus faulendem Fleisch und ähnliche allgemein anerkannte Phänomene auf eine blosse «eductio formarum ex potentia materiae» zurückzuführen. Die vielfältigen Vermögen und die komplizierte Struktur selbst einfacher, urgezeugter Lebewesen scheinen ihm unmöglich auf die Potenz elementarer Materie reduzierbar. In einem Teil der Fälle sei die Zeugung nur scheinbar spontan, weil, vom Beobachter unbemerkt, regelrechte Samenmaterie der entstehenden Art Zutritt gefunden habe ${ }^{39}$. Ansonsten muss bei Ausschluss einer äusseren Formenquelle auf eine Lösung zurückgegriffen werden, die der Erklärung der Arzneiwirkungen pflanzlicher und tierischer Stoffe ganz analog ist. Die Form des neuen, urgezeugten Lebewesens muss bereits zu Lebzeiten im Körper des mittlerweile verstorbenen, zuvor von einer höheren Form Beseelten, vorhanden gewesen sein ${ }^{40}$.

Dringlicher noch als in der Abhandlung der okkulten Qualitäten und ihres Ursprungs stellt sich damit erneut die Frage nach der Koexistenz nicht nur von mehreren Formen, sondern sogar von mehreren Seelen in einem Lebewesen. So sieht sich Sennert in der Interpretation der «generatio spontanea» zu einer weiteren Differenzierung seines Konzepts einer Formenhierarchie in den Lebewesen und übrigen Naturkörpern veranlasst. Mit Aristoteles sei zu sagen, dass alles in bestimmter Weise voll von Seele ist ${ }^{41}$. Das sei aber nicht so zu verstehen, dass alle Naturdinge beseelt seien, sondern Aristoteles habe sagen wollen,

«[...] dass in fast allen Dingen eine ungefähr so beschaffene Substanz ist, die sich, wo sie von allen Hemmnissen befreit auf eine geeignete Materie trifft, offenbart und im Amt der Seele fungiert.. ${ }^{42}$

Die Seele des urgezeugten Lebewesens sei schon im vorherigen Lebewesen durchaus «in actu», aber nicht als spezifische, sondern als dienende, untergeordnete Form. Deshalb dürfe man auch nicht meinen, dass im menschlichen Fleisch nicht nur die Formen von Würmern, sondern tatsächliche Würmer seien ${ }^{43}$. Erneut deutet er an, dass er diese zunächst noch unter- 
geordnete Form im «calidum innatum» verankert sieht ${ }^{44}$, doch anders als in De chymicorum [...] steht nun der Formcharakter gegenüber der Frage nach dem möglichen materiellen Substrat ganz im Vordergrund.

Genau besehen also entspricht die "generatio spontanea» in Sennerts Darstellung gar nicht mehr dem Werden eines neuen Lebewesens mit einer neuen Form, sondern der Herrschaftsübernahme durch eine längst existierende Form, deren Wirkvermögen bisher lediglich im Dienst der höheren Form standen. Die neue Form ist ebenso alt wie die vergehende. Beider Ursprung liegt im göttlichen Schöpfungsakt. Damit aber ist der naturphilosophische Sonderstatus der "generatio spontanea» weitestgehend aufgehoben. Sie folgt letztlich den gleichen Gesetzen wie die geschlechtliche Fortpflanzung: eine samenähnliche Materie unterwirft sich die sie umgebende Materie und teilt sich ihr formal mit. Es ist nur folgerichtig, dass Sennert der gleichen Materie die Fähigkeit zuschreibt, sich in der Folge dann auch wieder geschlechtlich fortzupflanzen.

\section{Atomistik}

An verschiedenen Stellen sind im bisher Ausgeführten schon die Vorzüge deutlich geworden, die Sennerts vielbeachtete Annahme einer korpuskulären Struktur der Materie für die Erklärung konkreter Naturphänomene bot. Sie erlaubte es, all jene Erscheinungen zu verstehen, bei denen kleinste oder gar unsichtbare Stoffmengen ihre komplexen Wirkkräfte dennoch integral bewahrten. Das galt für Gifte, stark zerkleinerte Arzneimittel und Kontagien, für die kleinsten Samenteilchen bei bloss scheinbarer Urzeugung, oder auch für die Laxantien und die Übertragung ihrer Wirkkräfte über die Muttermilch und überhaupt für die Nahrungsbestandteile und ihre Verteilung und Assimilation im Körper ${ }^{45}$. Die Bewahrung spezifischer Eigenschaften unter verschiedenen physikalischen Zuständen, etwa bei der Filterung von gelösten Stoffen durch feinstes Papier und ihrer anschliessenden Verfestigung, liess sich so schlüssig deuten. Die Annahme einer Teilchenstruktur der Materie erleichterte auch das Verständnis jener Vorgänge, die wir heute als Veränderung des Aggregatszustandes bezeichnen würden ${ }^{46}$. Die Verdunstung oder Verdampfung von Wasser war nicht mehr traditionell aristotelisch als eine Umwandlung des Elements Wasser in Luft zu verstehen ${ }^{47}$, was erneut die Frage nach der Herkunft der Form der Luft aufgeworfen hätte, 
sondern ganz analog als eine Verdichtung oder Verdünnung elementarer Teilchen, die dabei trotz des ganz unterschiedlichen Raumvolumens, das sie einnehmen, ihre Form bewahren ${ }^{48}$.

Sennerts Konzept, wonach die Naturkörper aus winzigen, von spezifischen elementaren oder überelementaren Formen bestimmten Teilchen aufgebaut sind, erweist sich also für die Deutung einer Vielzahl von Phänomenen als recht leistungsfähig. In der mehrfach zitierten Definition der «complexio» in Avicennas Canon medicinae konnte er sich zudem auf eine zentrale medizinische Autorität stützen, auf die schon Julius Cäsar Scaliger zurückgegriffen hatte. Danach ist die «complexio» das Ergebnis des AufeinanderEinwirkens der Qualitäten kleinster einander maximal berührender Teilchen ${ }^{49}$. Die angeführten Erscheinungen werden ihm ihrerseits zum Beleg für die Richtigkeit seines Erklärungsansatzes. Besonders gerne zitiert er das Beispiel der Steinablagerungen in Thermalbädern als anschaulichen Beweis für die Existenz kleinster Teilchen selbst im scheinbar völlig klaren Wasser $^{50}$.

Doch eine Korpuskulartheorie impliziert noch nicht die Annahme von echten Atomen, von unteilbaren Minima der Materie. Eben eine solche unterste Grenze der Teilbarkeit ist aber zentrales Charakteristikum der Sennertschen Teilchen. Hatte er den demokritischen Atomismus zunächst als unannehmbar reduktionistisch abgelehnt, so wandte er sich bald gegen Aristoteles, der in seiner Kritik an Demokrit den fundamentalen Unterschied zwischen den in der Tat unbegrenzt teilbaren mathematischen und den nur begrenzt teilbaren natürlichen Körpern verwischt habe ${ }^{51}$. Demokrit sei es nur um letztere gegangen, als er nach kleinsten, nicht mehr weiter teilbaren Körpern suchte, aus deren Zusammenkommen die natürlichen Körper entstünden ${ }^{52}$.

Im Kapitel «De atomis» des dritten Buches seiner Hypomnemata entwikkelt Sennert eine detaillierte Systematik der unterschiedlichen Arten von Atomen ${ }^{53}$. Zunächst sind da die Atome der Elemente. Aufgrund ihrer Kleinheit und Durchdringungsfähigkeit gestatten sie eine gänzlich unorthodoxe Deutung der Interaktion der Primärqualitäten. Die «alteratio», die Veränderung der Qualitäten eines Naturkörpers, erklärt sich nicht mehr traditionell aristotelisch aus der wechselseitigen actio und passio der Qualitäten, sondern sie ist das Ergebnis einer Ortsbewegung. Sie beruht auf dem Eindringen von Elementarteilchen, die somit mit ihren jeweiligen Qualitäten an dem Körper teilhaben ${ }^{54}$. Die Erwärmung von Wasser entspricht so dem Eindringen von Feuerteilchen, seine Abkühlung ihrer Entfernung. 
Über die Ebene der Elementaratome siedelt Sennert die der «prima mista» an, in welche die Körper bei den Fermentierungen, Verdauungen und Kochungen und damit auch bei den alchemistischen Verrichtungen aufgelöst und neu vereint würden. Über diesen schliesslich stehen die Atome der beseelten Körper, die zuweilen sogar eine vollständige Seele enthielten, was ja dann für die Deutung der «generatio spontanea» entscheidend sein sollte.

All diese unterschiedlichen Atome teilen Sennert zufolge die grundlegende Fähigkeit, ihre Form integral zu bewahren. Natürliche Körper benötigten aber für die Bewahrung ihrer Form ein gewisses natürlich vorgegebenes Minimum an Materie ${ }^{55}$. Genaugenommen ist damit eine weitere Teilbarkeit der Atome nicht ausgeschlossen. Sie verlören bei Unterschreiten einer bestimmten Mindestgrenze ihre Form, doch könnten zumindest bei den überelementaren Formen die bisher untergeordneten Formen die Herrschaft über die entstehenden kleineren Materiequanten übernehmen. Sennerts Interpretation lässt sich also am besten als eine Theorie der Form-Minima verstehen.

Die Frage nach den Motiven, die Sennert zur Annahme solcher echten Minima bewegten, ist damit freilich noch nicht zufriedenstellend beantwortet. Ein Grund ist sicherlich in Sennerts Beschäftigung mit der Diskussion um die «minima naturalia» zu suchen, mit der Frage also, ob es für die Naturkörper eine Grenze der Teilbarkeit gebe, unterhalb derer sie keinen Bestand haben ${ }^{56}$. Wiederholt zitiert er die 1613 erschienenen Optica des Aguilonius, welcher echte Minima des Lichts postuliert hatte, unterhalb derer das Licht nicht mehr weiter abgeschwächt werde, sondern gänzlich verlösche ${ }^{57}$. Gleiches, so Sennert, gelte für die Qualitäten und Wirkvermögen und mehr noch für die Formen selbst. Unterhalb einer bestimmten Grenze müssten sie untergehen, nicht aufgrund des Einwirkens entgegengesetzter Qualitäten, sondern einfach wegen des Fehlens eines Mindestmasses an materieller Konsistenz als einer naturgesetzlich vorgegebenen unabdinglichen Voraussetzung ihrer Selbsterhaltung ${ }^{58}$.

Sennerts Atomistik steht also in dieser jahrhundertealten Tradition, und er selbst gibt sich erstaunt ob der Unterstellung, er vertrete mit seiner Atomistik etwas Neues ${ }^{59}$. Die genauere Analyse lässt freilich noch einen uneingestandenen weiteren Grund für die Hinwendung zu einer echten Minima-Theorie - im Gegensatz zu einer blossen Teilchentheorie-vermuten, nämlich in den Argumentationsnöten, in die ihn der berühmt gewordene Häresie-Vorwurf des Groninger Professors Johannes Freitag brachte ${ }^{60}$. Dieser unterstellte Sennert, er habe die Unsterblichkeit der Tierseelen postuliert 
und damit die Einzigartigkeit der menschlichen Seele in Frage gestellt. Sennert konnte in Gutachten führender theologischer Fakultäten Entlastung von diesem Vorwurf finden ${ }^{61}$. Doch Freitag hatte nicht so ganz Unrecht, zumindest solange Sennert nicht ausdrücklich echte Minima der formbegabten Materie postulierte.

Hätte die Seele als die Form der Tiere in beliebig kleinen Quanten Bestand, so stellte sich in der Tat die Frage, wie sie dann überhaupt noch untergehen können soll. Die Überwältigung durch eine höhere Form kommt für die hochstehenden Tierseelen kaum in Frage und wäre in der Sennertschen Darstellung ohnehin nicht mit einer Vernichtung, sondern nur mit einer Unterordnung gleichbedeutend. Die Einwirkung übermächtiger Primärqualitäten mochte die Seele ihrer nötigen Instrumente berauben und die Verwirklichung jeglichen Wirkvermögens verhindern, aber im Rahmen der Sennertschen korpuskulären Mischungstheorie war das nicht gleichbedeutend mit der Vernichtung der Form und ihrer spezifischen Materie. Selbst wenn die Tierseele den sterbenden Körper mit einem spirituösen Etwas als ihrer spezifischen Materie verliesse, existierte sie dennoch weiter, selbst wenn sie kein neues Lebewesen ausformte. Der grundlegende Unterschied zwischen materiegebundener Tierseele und der als einziger nicht notwendig an Materie gebundenen unsterblichen Menschenseele wäre in der Tat aufgehoben. Auch die Tierseele wäre ewig. Nur wenn die spezifische Materie der Seele, das «calidum innatum», so weit zerteilt werden kann, dass eine natürliche Grenze erreicht wird, unterhalb derer sie den Fortbestand der Tierseele nicht mehr sichern kann, muss diese wirklich untergehen. Somit gewinnt Sennerts Annahme eines echten, quantitativ definierten FormMinimums hier tatsächlich zentrale Bedeutung. Die aus theologischer Sicht mindestens ebenso brisanten Implikationen von Sennerts streng immanentistischer Formenlehre, die Annahme einer materiell gebundenen Weitergabe und Vermehrung der individuellen Menschenseele über die Generationen, die Freitag nicht aufgegriffen hatte, bleiben davon freilich unberührt ${ }^{62}$.

\section{Schlussbemerkung}

Sennert, so lässt sich zusammenfassend sagen, legt einen beeindruckenden, wohldurchdachten und in vielen Punkten durchaus originellen naturphilosophischen Gesamtentwurf vor. Gewiss entspricht seine Sichtweise nicht mehr unserem heutigen veränderten Weltbild, obschon auch aus einer positi- 
vistischen Fortschrittsperspektive einzelne Thesen, etwa seine Annahme einer spezifischen, freilich als unveränderlich gedachten, über die Generationen weitergegebenen formbegabten Materie, die sich in der Embryogenese die umgebende Materie unterwirft und für ihre Zwecke anordnet, zumindest als glückliche Intuitionen erscheinen. Noch bedeutsamer erscheint jedoch Sennerts wissenschaftliche und erkenntnistheoretische Grundhaltung ${ }^{63}$. Seine Betonung des dynamischen Charakters der Formen und ihrer Erkennbarkeit, nicht über verstandesmässig abgeleitete Wesenheiten, sondern über ihre jeweiligen beobachtbaren Qualitäten und Wirkvermögen, sein gelassener Umgang mit den Lehrsätzen der Autoritäten, einschliesslich des von ihm verehrten Aristoteles, und sein Antireduktionismus, der den Menschen auf die empirische Untersuchung der naturgesetzlich gegebenen, fest in der Materie verankerten und je spezifischen Eigenarten und Wirkvermögen der Dinge verweist, verdeutlichen einmal mehr die Vielseitigkeit und Leistungsfähigkeit aristotelisierender Naturphilosophie in der Spätrenaissance. Mögliche Einflüsse auf die Weiterentwicklung der Philosophie in der Folgezeit zu denken wäre insbesondere an Leibniz - bedürfen noch der näheren Aufklärung. In der Auslegung, die Sennert ihr gibt, ist die Anwendung der Form-Materie-Lehre jedenfalls alles andere als ein Ausdruck theoretischer Erstarrung. Sie spiegelt allenfalls die Anerkennung der letztlich auf den unergründlichen göttlichen Schöpfungsakt zurückgeführten Komplexität der Natur und des Zusammenwirkens der Dinge im Rahmen einer ihnen innewohnenden, vom Menschen zu erforschenden Eigengesetzlichkeit*.

* Für kritische Anmerkungen zu einer ersten Fassung dieses Beitrags danke ich herzlich Herrn Prof. E. Kessler, München. 


\section{Anmerkungen}

1 Wittenberg 1611 : weitere, veränderte Auflagen Wittenberg 1620 und Wittenberg 1633.

2 Wittenberg $1619 ; 2$. Auflage Wittenberg 1629.

3 Vgl. W.Pagel: Paracelsus. An Introduction to Philosophical Medicine in the Era of the Renaissance. Basel-New York 1958, S.333-343; G.Zanier: Medicina e filosofia tra '500 e '600. Mailand 1983, besonders S. 100-103.

4 Hypomnemata physica. Orig. 1636. Hier zit. n. Opera omnia. 3 Bde. Venedig 1641, Bd.1, Proemium.

5 K. Lasswitz: Geschichte der Atomistik vom Mittelalter bis Newton, Bd. 1, Hamburg-Leipzig 1890, S.436-454; A.G. Van Melsen, From Atomos to Atom, New York 1960; G. B. Stones: The Atomic View of Matter in the XVth, XVIth and XVIIth Centuries. In: Isis 10 (1928), S. 445-465.

6 Den besten Überblick bieten derzeit T. Gregory: Studi sull'atomismo del seicento. In: Giornale critico della filosofia italiana 43 (1964), S. 38-65 und 45 (1966), S.44-65, mit einer Einordnung in die Atomistik des frühen 16. Jahrhunderts, und J. Roger: Les sciences de la vie dans la pensée française du XVIII' siècle. La génération des animaux de Descartes à l'Encyclopédie. Paris 1963, hier S. 106-112; s. a. den Beitrag zu Sennert von H. Kangro im Dictionary of Scientific Biography, New York 1975.

7 Vgl. beispielsweise das Vorwort (Lectori candido et benevolo) zu den Hypomnemata 1641 (unpaginiert): «divinae sapientiae infinitos thesauros in rerum Natura absconditos esse».

8 «Mihi si, quod sentio, liberè dicendum, vix vllam rei Physicae damnosiorem, \& quae potima ingenia irrito $\&$ inani labore magis fatigauerit, in Physicam irrepsisse puto opinionem, quam illorum, qui omnium eorum, quae in rebus naturalibus accidunt, causis è qualitatibus manifestis, \& quatuor elementis reddere conati sunt.» (Medicina practica In: Opera omnia 1641, Bd.3.) In diesem Zusammenhang macht Sennert auch wiederholt auf die Schwierigkeit aufmerksam, zu erklären, warum die Aktualisierung der Potenz zu einem gegebenen Zeitpunkt und nicht instantan, ohne Aufschub erfolgte.

9 «Non tamen [...] ex fortuito atomorum concursu res naturales fiunt [...], sed à forma superiore dirigente, quae Caloris instrumento \& ope attrahit, retinet, miscet, \& disponit omnia [...]» (De chymicorum [...] 1619, S.363).

10 Quaestiones medicae controversae septem [...], respondebit M. Michael Döring, Wittenberg 1607, Quaestio secunda.

11 «Essentia autem rei intendi atque remitti non potest...» (ebd. S. 13).

12 «... ita [in rebus naturalibus] non solum qualitates illae manifestae, caliditas, frigiditas, humiditas, siccitas, \& si quae alia sunt, reperiuntur, sed etiam nobiliores quaedam $\&$ occultiores, quae à formis illis nobilioribus fluunt $\&$ emanant, atque à quibus nobiliores $\&$ praestantiores actiones proficiscuntur. Atque ut illae mutuo se afficiunt: ita etiam inter occultas actionem quandam esse, negandum non est: cum ipsa id testetur experientia; quàm quidem occultarum qualitatum actionem vulgò à tota substantia nominant.» (ebd.) Zur Lehre von der «tota substantia» und zu Sennerts Krankheitsbegriff vgl. L. D. Richardson: The Generation of Disease: Occult Causes and Diseases of the Total Substance. In: The Medical Renaissance. Hg. v. A. Wear u.a. Cambridge 1985, S.175-194, und P. H. Niebyl: Sennert, Van Helmont and Medical Ontology. In: Bulletin of the History of Medicine 45 (1971), S.115-137. 
13 Quaestiones medicae controversae quinque [...], respondebit M. Ioachimus Koppe. Wittenberg 1607, Quaestio 3 : «Qua ratione pharmaca K $\alpha \vartheta \propto \varrho \tau \imath \kappa \alpha$ humores expurgent?»

14 «[...] temerarij esse judicamus, rerum abditissimarum arcana perscrutari, \& in naturam injurij pro veris occultis falsas manifestas reponere velle.» (ebd., Quaestio 3, Absatz 26)

15 «[...] rebus omnibus proprietates quasdam nobiliores inditas esse, quibus quod sibi proprium est agunt, pro forma natura \& motione insita [...]» (ebd., Quaestio 3, Absatz 25). Ähnliche Auffassungen finden sich schon bei I.Schegk (Tractationum physicarum et medicarum tomus unus, Frankfurt 1585), der die alten Begriffe der «forma specifica» und der Wirkung «kat' entelecheian» verwendet.

16 Im Kapitel «De morbis totius substantiae seu occultarum qualitatum» (S. 131-145) findet sich dort auch eine noch ausführlichere Abhandlung des «tota substantia»-Konzepts.

17 «Neque enim qualitates primae in minimo corpore summas vires exercere possunt.» (Institutiones 1611, S.242). Sennert zitiert Galens De simplicium medicamentorum facultatibus, Lib. 5, c. 19: «Sunt quae tota substantia nobis sunt contraria. [...] Nam talia omnia genere sint deleteria, non quantitate.» (ebd.)

18 Ebd.

19 «[...] quod communicatur, est quiddam praeter naturam, $[\ldots]$ corpus quoddam \& semen quasi $[\ldots]$ Ad hujus autem seminis naturam pertinet, ut qualitatem illam occultam, qua morbum à tota substantia dictum in corpore, à quo recipitur, excitare possit, obtineat : ob quam etiam in exiguo corpore magna contagii vis est. Cum enim elementares qualitates, ut multum agant, etiam multam desiderant materiam: hae qualitates, quia formae asseclae sint, etiam in exiguo quanto magnam habent vim, atque ad inficiendum totum corpus minima quantitas talis seminis sufficit.» (ebd., S. 248)

20 Ebd., S. 250-252 und S.807.

21 De chymicorum [...] 1619, S. 248 f.; Hypomnemata 1641, S. 14.

22 Zur «colchodea» vgl. B. Nardi: Origine dell'anima umana. In: ders.: Studi su Pietro Pomponazzi, Florenz 1965, S.231-246, S. 234 f., Fussn.

23 Allgemein zur aristotelischen Naturphilosophie vgl. F. Solmsen: Aristotle's System of the Physical World. Ithaca-New York 1960; I. During: Aristoteles. Darstellung und Interpretation seines Denkens. Heidelberg 1966.

24 Sennert beruft sich dabei auf Iacobus Zabarella und dessen Theorie, wonach die Form des Gemischten nach dem Untergang der Seele ihrerseits das «officium» der spezifischen Form übernehme, der sie zuvor als Materie gedient habe. (Hypomnemata 1641, S. 73; die Stelle bei Zabarella ist: De rebus naturalibus libri XXX, 4. Ausg. Köln 1602, S. 395-399, Liber de communi rerum generatione).

25 «Quae quidem formae per se consideratae etiam verae formae sunt, quae materias suas informant: sed respectu animae $\&$ formae specificae rationem materiae immediatiae habent.» (Hypomnemata 1641, S. 14)

26 «[...] remanent aliquandiu hae formae, \& formae officio funguntur, \& facultatum atque operationum, quae in rebus pluribus amplius non viventibus deprehenduntur, neque tamen ex elementorum qualitatibus vel formis provenire possunt, authores sunt.» (ebd.)

27 «Constat autem calidum innatum spiritu insito \& humido primigenio : estque calidum innatum humidum primigenium insito spiritu \& calore undique perfusum. (Epitome naturalis scientuae. Wittenberg 1618, S.605). Zur Geschichte der Lehre vom «calidum innatum» vgl. E. Mendelsohn: Heat and Life. The Development of the Theory of Animal Heat. 
Cambridge 1964; F.Solmsen: The Vital Heat, the Inborn Pneuma, and the Aether. In: Journal of Hellenic Studies 77 (1957), S.119-123.

28 «Etsi ignis in mistis utpote temperatus, alio modo agat, quam simplex: tamen nec tum ultra suas vires agit. Et nihil praestat nisi quod in sua \& reliquorum, cum quibus mistus est, elementorum potestate est.» (De chymicorum [ . . ] 1619, S.247f.)

29 Epitome 1618, S.605.

30 Mit diesem Thema hatte sich Sennert schon früh auseinandergesetzt; vgl. J. Jessenius von Jessen: De sympathiae et antipathiae rerum naturalium caussis disquisitio singularis, quam in publico pro virili ad Cal. Iunij defendere conabitur M. Daniel Sennertvs Vratislaviensis. Wittenberg 1599.

31 «[...] cum multis doctis viris, Fernelio, Valeriola, Schegkio, \& alijs plurimis probabiliter asserimus: Esse quid spirituosum in rebus naturalibus praeter elementa» (De chymicorum [...] 1619, S.260). Sennert versäumt nicht, auf die aristotelische Charakterisierung des «spiritus» als «aitheroides», als der Materie der Sterne ähnlich, hinzuweisen (Hypomnemata 1641. S.8).

32 «Neque sola caliditas est instrumentum animae: sed totum illud corpus, quod calidi vel spiritus insiti nomine venit, \& non solum calore, sed \& aliis nobilioribus qualitatibus instructum est.» (Institutiones 1620, S.35 f.)

33 De chymicorum [...] 1619, S.257-259. Später verwendet Sennert die Begriffe «spiritus» und «calidum innatum» wieder zurückhaltender und führt die okkulten Qualitäten der Gemmen, Steine und Mineralien allgemeiner auf deren überelementare spezifische Form zurück; selbst den Pflanzen kommt in den Hypomnemata nur mehr ein «calidum innatum»-Analogon zu (Hypomnemata 1641, S. $13 \mathrm{f}$.).

34 «Si enim coctione vel infusione ea substantiae pars, in qua vis purgandi est, separetur \& extrahetur, vtile medicamentum paratur; reliquum verò corpus planè inefficax relinquitur.» (Hypomnemata 1641, S. 14.)

35 «Rectius, vt dictum, sacrae literae nos docent, in principio elementa creata esse, $\&$ res omnes $[\ldots]$ formas, quibus à se inuicem differunt \& efficaces sunt, ex prima creatione habere, quasque Dei Opt. Max. voluntate ad hunc isque diem conseruant.» (Hypomnemata 1641, S.4)

36 «Creator enim rerum generationis fundamentum posuit seminium cujusq; rei, per quod generabilia propagantur, ac ipse primitus totum miscuit; atque ita in rerum generatione hoc, quod ab initio rebus inditum corpus, in generatione propagatur, \& ab ijs, quae primò condita sunt, principijs adhuc hodie constituitur, regitur \& conservatur.» (De chymicorum [...] 1619, S.261.)

37 Hypomnemata 1641, S.65-97.

38 Zur Vorgeschichte dieser Lehre vgl. E. Lesky: Die Zeugungs- und Vererbungslehren der Antike und ihr Nachwirken, Mainz 1950.

39 Hypomnemata 1641, S. 72.

40 Wie aus pflanzlichem Holz tierische Würmer, denen doch eigentlich eine höhere Form zukommen müsste, spontan gezeugt werden sollen, lässt Sennert freilich offen.

41 ««quoddammodo animarum plena sunt omnia»» (ebd., S. 71).

42 «[...] quòd in omnibus ferè rebus sit talis ferè substantia, quae, vbi omnibus impedimentis remotis idoneam materiam nacta est, sese exserit, $\&$ animae officio fungitur.» (ebd., S. 71.) 
Sennert führt hier auch seine Lieblingsautorität J.C.Scaliger an, der die «generatio spontanea» auf eine samenähnliche Materie zurückgeführt habe (ebd., S. 73). Zu Scaliger vgl. Van Melsen [wie Anm. 5], S. 73-77.

43 Ebd., S.73.

44 «[...] anima subiectum illud habet, in quo erat in corpore vivente, dum superiori formae loco materia proxima esset.» (Hypomnemata 1641, S.76)

45 Hypomnemata 1641, $20 \mathrm{f}$.

46 Hypomnemata 1641, S. 21.

47 Diese Auffassung hatte 1618 auch Sennert noch vertreten (Epitome 1618, S. 232).

48 Hypomnemata 1641, S.76.

49 Sennert zitiert Avicennas Definition der «complexio» als «qualitas, quae ex mutua actione \& passione contrariarum qualitatum elementorum quorum partes ad minimas redactae, ut cuiusque earum plurimum contingat plurimum alterius, provenit.» (Zit. nach Institutiones 1611, S.15.)

50 De chymicorum $[\ldots]$ 1619, S. 362 . In analoger Weise hatte er schon lange vor Entwicklung seiner Atomistik die Entstehung von Nieren- und Blasensteinen im Körper als allmähliche Ab- und Anlagerung kleinster Salzteilchen erklärt, die in den Körperflüssigkeiten als solche nicht wahrnehmbar seien (Quaestiones medicae controversae quinque [...] respondebit M. Donatus Freywaldus. Wittenberg 1608, Quaestio 2).

51 De chymicorum [...] 1619, S. 359; Hypomnemata 1641, S. 17.

52 Hypomnemata 1641, S.17.

53 Hypomnemata 1641, S.16-22.

54 «[...] alterationes vulgò dictas non esse saltem in qualitatibus mutationes, sed corporis alieni partecipationem» (Hypomnemata 1641, S. 19).

55 «Ita enim cum rebus naturalibus comparatum est, vt formae non nisi certo quanto consistant; \& sicut vltrà naturales terminos sese non diffundere: ita non nisi in certo quanto conseruari possunt.» (Hypomnemata 1641, S.22)

56 Grundlegend: A.Maier: Studien zur Naturphilosophie der Spätscholastik. Rom 1949, S. 155-188.

57 F. Aguilonius: Opticorum libri sex Philosophis iuxtà ac Mathematicis utiles. Antwerpen 1613, S. 379, Lib. 5, propositio 8.

58 «Quo pacto \& corporum naturalium minima dantur, quae nimirum, si amplius dividuntur, formam essentiamque deperdunt.» Und zwar vergingen sie nicht aufgrund der Einwirkung entgegengesetzter Qualitäten, «[...] sed solius mensurae defectu, quae ad propriam cuiusque tutelam velut necessaria conditio irrefragabili naturae lege expositur.» (Hypomnemata 1641, S. 22)

59 Hypomnemata 1641, S.17.

60 P.Bayle: Dictionnaire historique et critique. Paris 1820-1824, Repr. Genf 1969; s.a. W.-U.Eckart: Der Streit zwischen Daniel Sennert (1572-1637) und Johann Freitag (1581-1641). In: Deutsch-Niederländisches Medizinhistorikertreffen. Vorträge. Münster 1978, S. 21-38.

61 De origine et natura animarum in brutis. In: Opera omnia. 3 Bde. Lyon 1676. Bd.1, S. 285-306.

62 Die Hypomnemata wurden schon bald nach ihrem Erscheinen auf den Index gesetzt. Als mögliches Motiv deutet P. Redondi die theologisch brisanten Implikationen der atomisti- 
schen Materielehre für die Deutung der Transsubstantiation in der Eucharistie an, die er auch für Galileis Verurteilung für entscheidend hält (P. Redondi : Galileo der Ketzer. München 1990).

63 Einige Hinweise hierzu auch bei W.-U. Eckart: Grundlagen des Medizinisch-Wissenschaftlichen Erkennens bei Daniel Sennert (1572-1637) untersucht an seiner Schrift «De chymicorum ...liber», Wittenberg 1629. Diss. med. Münster 1978.

PD Dr. med. Michael Stolberg

Institut für Geschichte der Medizin

und Medizinische Soziologie der TU München

Ismaninger Strasse 22

D-8000 München 80 